\title{
Teixobactin: A New Class of Antibiotic
}

\author{
Tim Sandle* \\ Head of Microbiology, Bio Products Laboratory Limited, Elstree, UK
}

Received: January 12, 2015; Accepted: January 12, 2015; Published: February 12, 2015

*Corresponding author: Tim Sandle, Head of Microbiology, Bio Products Laboratory Limited, 68 Alexandra Road, London Colney, St. Albans Hertfordshire, UK, Tel: +07-808-906409; E-mail: timsandle@btinternet.com

\section{Editorial}

A new antibiotic has been discovered: Teixobactin. This in itself represents a significant breakthrough since it is the first new type of antibiotic to be discovered since the late 1980s. The discovery carries more significance especially in the era of antibiotic crisis, in which a rising number of microbial strains are evolving resistance to common antibiotic (and in the case of a narrower but more potent range, "multi-drug resistant" which infers resistance to more than one type of antibiotic.)

Though we welcome the new antibiotic, their discovery reports are addressed in much of the popular media (and, unfortunately, in some of the scientific press) are always tainted by hyperbole. (And, unfortunately, in some of the scientific press) is tainted with hyperbole.

The antibiotic crisis is the greatest threat, within the field of science and medicine, faced by humanity. Since Alexander Fleming's discovery in 1929 [1] and with the industrial scale production of antibiotics from the 1940s, humanity has benefited from a spectrum of natural and synthetic antimicrobial compounds. These chemicals have enabled people to go to hospital and undergo an operation with minimized risks of cross-contamination from bacteria (from the environment or the improperly sanitized hands of surgeons). In most cases of bacterial infections the risk were lowered and rapidly cleared up by giving a short course of antibiotics [2]. Therefore, in this $20^{\text {th }}$ century, antibiotics have helped to reduce the risk of potentially life threatening complications following surgery, chemotherapy and transplantation [3].

The antibiotic crisis has arisen due to bacterial evolution and plasmid transfer, where genetic modifications can impart resistance. One such situation is where pathogenic microbes continue to grow after exposure to one or more antimicrobial agents. In that case the strain becomes resistant and becomes wide and spread within a community, an alternative antibiotic needs to be administered. The risk is then that a bacterium becomes resistant to multiple antibiotics and theoretically this process would continue until there are no effective antibiotics. Currently, carbapenems stand as the class of chemicals that are frequently used as antibiotics of last resort.

This process of antibiotic resistance has accelerated through
Over-use of antibiotics (although, arguably, acquired resistance is eventually inevitable). Over-use has occurred through medics handing antibiotics for no good reason (a common case here is for people who have viral infections like the common cold or influenza being mis-prescribed).

The so-termed "age of the superbug", although genetically inevitable, need not have affected society to the extent that it has. The reason why no new antibiotics have emerged for nearly thirty years is not because it is beyond human ingenuity to create synthetic ones or we have exhausted the hunt through all of the microbial species of the world. Many antibiotics are derived from other microbes, the classic example being penicillin which is a group of antibiotics derived from Penicillium fungi.

The reason why new antibiotics have not emerged is because of pharmaceutical companies went through a period of less research for developing them, the reason being very low return on investment that such medications command in the market place. However this situation changed a little a few years ago when governments undertook, research as a result of antibiotic shortage crisis, to incentivize pharmaceutical corporations to undertake measures (see, for instance, U.S. Senator Sherrod Brown's Strategies to Address Antimicrobial Resistance (STAAR) Act.) Nonetheless, most of the new research, including teixobactin, has arisen from the university sector (although there has always been a relationship between universities and industry since the private sector is a key source of funding).

The main reason for antibiotic resistance is due to some farmers, who are engaged in intensive farming methods gave antibiotics to their animal's in order to boost the meat quantity.

The newly reported antibiotic teixobactin was isolated from the so-termed microbial "dark matter" (to draw on terminology commonplace in physics). This bacterial species that cannot be cultured in the laboratory are termed as "viable but nonculturable" microorganisms. These are bacterial cells that do not grow on plate media but retain their viability. Some organisms are culturable and have the ability to grow in the medium whereas few organisms are non culturable and do not have the ability to grow in the medium. These organisms will cause severe infections to humans [4].

The discovery of the new antibiotic was made by the U.S. 
scientist from North- eastern university, Boston. He is also working in Novo Biotic Pharmaceuticals. The research has been published in the journal Nature [5].

Scientists' unearthed teixobactin from a soil-dwelling bacterium termed Eleftheria terrae. Due to unculturable nature the isolated antibiotic-producing bacteria were allowed to grow in a chamber within the soil for further study. For this they constructed a specially designed chamber, dubbed iChip. With this novel technique, a soil sample was collected and diluted with agar. A single bacterial cell is suspended in a chamber surrounded with semi-permeable membrane. 96 of these chambers were then placed into a single device. The device was then lowered into the soil. This allows bacteria to access nutrients and other growth factors. The discovery was not an easy process; the researchers screened some 10,000 bacterial strains before identifying twenty-five potential candidates. The most promising organism was found underneath the grass in a field in Maine, U.S.A.

The new antibiotic has shown potent activity against Gram-positive bacteria, with no signs of resistance. It can kill a range of bacterial species, as demonstrated in mouse studies, including Methicillin-Resistant Staphylococcus aureus (MRSA) and Mycobacterium tuberculosis. The discovery of teixobactin also demonstrates that there are compounds that may have exceedingly low probability of resistance, providing researchers with a new strategy for antibiotic development. Some bacteria that need to be sourced will not grow in the laboratory culture media.

Whilst teixobactin represents a significant step forwards, it is important to pause and consider what exactly the antibiotic can do and what its limitations are.

First, it is a new class of antibiotic (class refers to the mechanism by which it inactivates or destroys a bacterial cell). This is not, the only antibiotic to be discovered and given approval recently. In 2014, two compounds were approved by the U.S. Food and Drug Administration (FDA) [6]. These are Dalvance, which is an intravenous drug that can treat skin and soft tissue infections. Dalvance is intended to treat acute bacterial skin and skin structure infections and the second antibiotic was Sivextro or, tedizolid phosphate (non-commercial name). Those antibiotics which are reviewed on the digital platform have been approved for intravenous or oral use to treat severe MRSA. Other research projects were carried out using gene editing, among other research projects, to stop bacteria in their tracks.

Second, there is an issue regarding spectrum of activity.
All the listed bacteria in relation to the destructive properties of teixobactin are Gram-positive. There is no Gram- negative bacteria like pseudomonas or Klebsiella, because teixobactin is not effective against them. Teixobactin is extracted from a type of Gram-negative bacterium (E. terrae.) The antibiotic cannot, naturally, be effective against Gram-negative microorganisms; otherwise the bacterium would inhibit its own growth through extruding the antibiotic chemical.

Third, the antibiotic is still a long way from the clinic, and has to undergo a series of rigorous human clinical trials before reaching the pharmacy shelves. This will be a matter of years, not months.

Fourth, the mainstream media have presented the antibiotic as being somehow super-resistant, as if to imply that no bacterium in the future will be able to develop resistance against it. Although the compound targets lipid molecules, which guards against the rapid development of resistance, prevailing microbiological theory stands that any bacterial species will develop resistance in time. This is why the search for new antibiotics needs to be continuous. In relation to this, the study notes that the new antibiotic has a similar potential for resistance to vancomycin. The concern here about the evidence towards vancomycin resistance by bacteria which will be possible within next few years.

Thus teixobactin are a step forward. However, it should not be heralded that the answer to all medicines are based on the bacterial concerns. Far more research is needed and far greater prudence on the part of farmers, medics and patients is required in limiting the use of antibiotics to only serious relevant infections.

\section{References}

1. Fleming A. On the antibacterial action of cultures of a Penicillium, with special reference to their use in the isolation of B. influenza. Br. J. Exp. Pathol. 1929; 10(3): 226-236.

2. Sandle T. Taking on the Resistance, Laboratory News. 2014; 8-10.

3. Sandle, T. Taking on the Resistance, Laboratory News. 2014; 8-10.

4. Roszak DB, Colwell RR. Survival strategies of bacteria in the natural environment. Microbiol Rev. 1987; 51(3): 365-379.

5. Ling L L, Schneider T, Peoples A J, Spoering AL, Engels I, Conlon BP, et al. A new antibiotic kills pathogens without detectable resistance. Nature. 2015; 517(7535): 455-459. doi: 10.1038/nature14098.

6. Sandle T. Novel Methods to Address Antimicrobial Resistance. SOJ Microbiol Infect Dis. 2014; 2(1): 2-3. 